\title{
Cerebral Venous Drainage in Patients With Space-Occupying Middle Cerebral Artery Infarction: Effects on Functional Outcome After Hemicraniectomy
}

\section{OPEN ACCESS \\ Edited by: \\ David S. Liebeskind, University of California, Los Angeles, United States \\ Reviewed by: \\ Anne-Katrin Giese, \\ Harvard Medical School, \\ United States \\ Saadat Kamran, \\ Hamad Medical Corporation, Qatar \\ *Correspondence: \\ Hauke Schneider \\ hauke.schneider@ \\ uniklinikum-dresden.de \\ tThese authors have contributed equally to this work \\ $\neq$ Johannes C. Gerber orcid.org/0000-0001-7465-8700 \\ Matthias Kuhn \\ orcid.org/0000-0003-2868-5155 \\ Hauke Schneider \\ orcid.org/0000-0002-9641-0922}

Specialty section:

This article was submitted to

Stroke,

a section of the journal

Frontiers in Neurology

Received: 08 May 2018 Accepted: 27 September 2018

Published: 17 October 2018

Citation:

Puetz V, Gerber JC, Krüger P, Kuhn M, Reichmann H and Schneider H (2018) Cerebral Venous Drainage in Patients With Space-Occupying Middle Cerebral Artery Infarction: Effects on

Functional Outcome After Hemicraniectomy

Front. Neurol. 9:876.

doi: 10.3389/fneur.2018.00876

\author{
Volker Puetz ${ }^{1 \dagger}$, Johannes C. Gerber ${ }^{1+\neq}$, Philipp Krüger ${ }^{2}$, Matthias Kuhn $^{3 \neq}$, \\ Heinz Reichmann ${ }^{1}$ and Hauke Schneider ${ }^{1,4 * \neq}$ \\ ${ }^{1}$ Department of Neurology, University Hospital Carl Gustav Carus, Technische Universität Dresden, Dresden, Germany, \\ ${ }^{2}$ Department of Anesthesiology, Klinikum Dortmund gGmbH, Dortmund, Germany, ${ }^{3}$ Carl Gustav Carus Faculty of Medicine, \\ Institute for Medical Informatics and Biometry, Technische Universität Dresden, Dresden, Germany, ${ }^{4}$ Department of \\ Neurology, Klinikum Augsburg, Augsburg, Germany
}

Background: Cerebral venous drainage might influence brain edema characteristics and functional outcome of patients with severe ischemic stroke. The purpose of the study was to evaluate whether hypoplasia of transverse sinuses or the internal jugular veins is associated with poor functional outcome in patients with space-occupying middle cerebral artery (MCA) infarction who underwent decompressive surgery.

Methods: We performed a retrospective analysis of patients with space-occupying MCA infarction treated with decompressive surgery at our university hospital. The transverse sinuses and the internal jugular veins were evaluated on baseline images and categorized as normal, hypoplastic or occluded. We defined composite variables for ipsilateral, contralateral or any abnormal cerebral venous drainage. We assessed the functional outcome at 12 months with the modified Rankin scale (mRS) score and defined poor functional outcome as mRS scores 5 and 6 .

Results: We analyzed 88 patients with available baseline imaging data [mean [SD] patient age $53( \pm 9)$ years; median[IQR] time to decompressive surgery 31(22-51) h]. At 12 months 44 patients (50\%) had a poor outcome. In univariate analysis neither ipsilateral (OR 1.98;95\%Cl: 0.75-5.40), nor contralateral (OR 1.56;95\%Cl: 0.59-4.24) or any (OR 1.6; $95 \% \mathrm{Cl}$ : 0.68-3.79) hypoplasia or occlusion of venous drainage were significantly associated with poor functional outcome. In multivariate analyses, higher patient age (OR 1.07;95\% Cl 1.01-1.14) and baseline stroke severity (OR 3.42;95\% Cl 1.31-9.40) were independent predictors of poor functional outcome, but not ipsilateral hypoplasia or occlusion of venous drainage (OR 1.31;95\% Cl 0.47-3.67).

Conclusions: The cerebral venous drainage pattern was not significantly associated with poor functional outcome in our cohort of patients with space-occupying MCA infarction who underwent decompressive surgery.

Keywords: stroke, middle cerebral artery infarction, space-occupying infarction, decompressive surgery, hemicraniectomy, cerebral venous drainage, cerebral sinus, cerebral veins 


\section{INTRODUCTION}

Space-occupying MCA infarction occurs in up to $10 \%$ of patients with supratentorial ischemic stroke (1). Brain edema formation of ischemic tissue exerts a mass effect with impending cerebral herniation and early clinical deterioration (2). Early decompressive surgery improves survival rates and functional outcome of patients with large MCA infarction (3-7). Conservative treatment strategies, e.g., osmotherapy, barbiturate sedation, or hypothermia, are less effective or without proven clinical benefit, respectively (8-10).

Space-occupying brain edema usually arises within 2 to 5 days after stroke onset, but the extent of brain edema varies (8). Previously, an abnormal ipsilateral cerebral venous drainage (CVD) has been shown to be associated with the development of fatal brain edema after internal carotid artery (ICA) or proximal MCA occlusion in a case series including 14 patients (11). In this series, 4 of 5 patients with a malignant edema had an abnormal ipsilateral CVD characterized by hypoplasia or occlusion of the transverse sinus or the internal jugular vein, whereas all 9 patients without malignant edema had normal ipsilateral CVD.

Factors affecting the clinical course and functional outcome after early decompressive surgery are not well understood. Prospectively acquired data on the effect of timing of surgery on clinical outcome are inconclusive (8). The impact of CVD on the development of infarct edema and the clinical course after decompressive surgery for space-occupying MCA infarction is unclear.

The aim of our study was to analyze the association of cerebral venous drainage patterns with the clinical course and functional outcome of patients with space-occupying MCA infarction after decompressive surgery. Our main hypothesis was that impaired venous drainage ipsilateral to the side of the infarction would result in edema expansion and subsequent poor functional outcome despite decompressive surgery.

\section{METHODS}

\section{Study Design and Patient Selection}

We performed a retrospective, single-center cohort study and used a local bone storage database to identify stroke patients aged 18 years and older with space-occupying MCA infarction who underwent decompressive surgery at our university hospital between 2001 and 2010. All patients with available baseline imaging datasets prior to hemicraniectomy [cerebral non-contrast CT (NCCT), CT angiography (CTA), magnetic resonance imaging (MRI), MRI angiography (MRA) and/or digital subtraction angiography (DSA)] were included into the analysis. Patients with missing clinical follow-up data were excluded.

\section{Stroke Treatment and Critical Care}

Patients were monitored and treated according to current European guidelines for the management of acute ischemic stroke and according to our institutional standard for treatment of space-occupying MCA infarction as follows: Decompressive surgery and ipsilateral intracranial pressure probe insertion was performed if patients had clinical signs of large MCA infarction, showed impaired consciousness, were 18 years or older, and if imaging revealed an infarction of $>50 \%$ of the MCA territory. Postoperatively, all patients were treated at our neurocritical care unit. Therapeutic hypothermia was performed with a target core temperature of $33-34^{\circ} \mathrm{C}$ for $96 \mathrm{~h}$ if no contraindications were present. Further critical care measures were reported elsewhere (10). Briefly, sustained elevated intracranial pressure (>15 mmHg) and cerebral perfusion pressure below $70 \mathrm{mmHg}$ were treated with osmotherapy (mainly mannitol $20 \%$ as short infusion every 4 to $6 \mathrm{~h}$ ) and/or with vasopressors, respectively.

\section{Data Retrieval and Management}

Baseline parameters and treatment measures were retrieved from our electronic patient management systems $\left(\mathrm{ICM}^{\circledR}\right.$, Dräger, Germany; ORBIS ${ }^{\circledR}$, AGFA Healthcare, Germany) or paper patient files. Functional outcome at 12 months was evaluated retrospectively using structured telephone interviews and mailed questionnaires including the structured assessment of the modified Rankin scale sore. Discharge letters of rehabilitation centers and entries of registers of residents were used to verify outcome data including date of death.

\section{Image Analyses}

Analyses of imaging data was performed as a three reader consensus by two stroke neurologists (V.P., H.S.) and one neuroradiologist (J.C.G.), blinded for clinical and outcome data of individual patients. We evaluated the cerebral venous drainage via the transverse sinuses and the internal jugular veins in regard to the side of the MCA infarction and classified it into (1) occlusion (or aplasia), (2) hypoplasia, (3) normal appearance, and (4) non-availability due to technical reasons (e.g., CT scan level). Composite variables were defined for venous drainage: (A) ipsilateral, (B) contralateral, or (C) any occlusion/hypoplasia of internal jugular veins or transverse sinuses. Images acquired before hemicraniectomy were analyzed for the extent of MCA infarction using the Alberta Stroke Program Early CT Score (ASPECTS) and for the presence of additional infarction in vascular territories not represented by ASPECTS $(12,13)$. Postoperative images were analyzed for the size of hemicraniectomy (maximum outer diameter on NCCT). Imaging modalities were cerebral NCCT, CTA, MRI, MRA, and/or DSA, depending on availability. In patients where only NCCT or MRI was available, we diagnosed hypoplasia or occlusion of transverse sinus if the associated bony indentation was layed out less than $50 \%$ compared to the opposite side.

\section{Statistical Analyses}

Clinical and neuroimaging characteristics were compared between patients with poor long-term functional outcome (mRS 5-6 at 12 months) and patients with mRS 0-4 at 12 months. For group-comparisons data are presented as mean (standard deviation) and compared using the Student- $T$-test, or as median (interquartile range, IQR) and compared using the Mann-Whitney $U$-test. Frequency distributions were analyzed by Pearson's chi-square and Fisher's exact test (two-sided). Statistical significance was defined as $p<0.05$ for all tests. 
To assess the effect of venous drainage patterns on longterm functional outcome we used multiple logistic regressions to model the probability for poor outcome while adjusting for other important clinical factors which were significant predictors of outcome in univariate analysis or considered clinically relevant a priori. The statistical AIC-criterion guided us in model evaluation. Likelihood ratio tests were used to assess statistical significance for predictors in the logistic regression. The goodness of fit of different models was evaluated based on residual plots and the Hosmer-Lemeshow test.

We performed statistical analyses using the software packages SPSS 23.0 and R 3.3.

\section{Approvals and Consent}

The study protocol was approved by the ethics committee "Ethikkommission an der Technischen Universität Dresden," Dresden, Germany (IRB00001473; EK125042012). Written informed consent was obtained by patients or their legal representatives in accordance with the Declaration of Helsinki.

\section{RESULTS}

\section{Patients}

We identified 108 stroke patients with space-occupying MCA infarction who were treated with decompressive surgery at our university stroke center. Eighty eight patients with available imaging data and follow-up data were included into the study (Figure 1). Imaging data were rarely available for patients of the early study period.

Demographic and clinical characteristics, treatment course, and outcome are summarized in Table 1. Patients were predominantly male (64\%), mean age was 53 years, and the dominant hemisphere was affected in $53 \%$ of patients. Median time from symptom onset to decompressive surgery was $31 \mathrm{~h}$. Most patients received treatment with osmotherapy $(n=63)$ and/or hypothermia $(n=74)$.

Twenty of 88 patients died during intensive care unit stay (cerebral herniation, $n=14$; medical complications, $n=5$; hematoma after hemicraniectomy, $n=1$ ). At 12 months after stroke onset, $50 \%$ of the patients had a mRS score of $0-4$ and 30 (34\%) patients had died.

\section{Baseline Imaging Data and Venous Drainage Pattern}

Pre-operative cerebral imaging revealed a median (IQR) ASPECT score of 0 (0-2), an additional anterior cerebral artery (ACA) infarction in $24 \%$ and an additional posterior cerebral artery (PCA) infarction in $11.4 \%$ of patients (Table 2). The median (IQR) diameter of hemicraniectomy was 121 (115-130) millimeters.

For the analysis of the venous drainage pattern we used cerebral imaging including CTA or DSA in $67 \%$ of the patients and NCCT or MRI in the remaining patients (Table 2, Figure 2). Image analysis of CVD patterns revealed a hypoplasia or occlusion in one or more of the evaluated venous segments in 50 of 88 patients (57\%) and in 84 of $312(30 \%)$ venous segments evaluated (Table 2). Hypoplasia or occlusion ipsilateral to the side of the infarction was found for the transverse sinus in 26 of 88 (30\%) patients and for the internal jugular vein in 16 of 88 (18\%) patients. A contralateral hypoplasia or occlusion was found for the transverse sinus in $30 \%$ and for the internal jugular vein in $18 \%$ of patients. Normal appearing transverse sinus and internal jugular vein were present in 59 and $71 \%$ of patients on the ipsilateral and contralateral side to the infarction, respectively.

\section{Group Comparison of Patients With Poor Outcome at 12 Months (mRS 5 and 6) and Patients With mRS 0-4}

Apart from higher baseline NIHSS scores and lower age, demographic factors and treatment characteristics were comparable in patients with good (mRS score 0-4) and poor (mRS score 5-6) functional outcome (Table 1). The extent of baseline MCA ischemia as quantified with ASPECTS was not different between both groups, but an additional infarction of the ACA and/or PCA territory was more frequently present in patients with poor outcome (Table 2).

The ipsilateral transverse sinus tended to be more often occluded or hypoplastic in patients with poor outcome compared to patients with good functional outcome [15/44(34\%) vs. 10/44 (23\%), respectively; $p=0.275]$. The rate of venous occlusion or hypoplasia of the ipsilateral internal jugular vein was comparable in patients who had a poor outcome compared to patients with a favorable outcome [9/44(21\%) vs. 7/44(16\%), respectively; $p=$ $0.799)$. Occlusion or hypoplasia of the contralateral transverse sinus [12/44(27\%) vs. $14 / 44(32 \%)$, respectively; $p=0.576]$, or the contralateral internal jugular vein $[8 / 44$ (18\%) vs. 8/44 (18\%)] were distributed similarly between patients with poor outcome compared to patients with good functional outcome.

\section{Association of Abnormal Cerebral Venous Drainage With Functional Outcome at 12 Months}

In univariate analyses, abnormal CVD patterns showed nonsignificant associations with poor functional outcome at 12 months: ipsilateral abnormal CVD (i.e., hypoplasia or occlusion of internal jugular vein or transverse sinus), Odds ratio [OR] 1.98 (95\%CI 0.75-5.40); contralateral abnormal CVD, OR 1.56 (95\%CI 0.59-4.24); any abnormal CVD (ipsilateral and/or contralateral), OR 1.6 (95\%CI 0.68-3.79). When any impaired CVD was defined as numeric variable $(0,1$, or 2 venous vessels with impaired drainage), univariate analysis showed an increased probability for poor outcome for patients with higher numbers of venous vessels affected (OR 1.76, 95\%CI 0.8-4.0), again not statistically significant.

Of other factors tested as potential predictors for poor functional outcome, only higher NIHSS score on admission ( $p=$ $0.004)$, higher patient age $(p<0.017)$ and additional infarction of ACA and/or PCA territory $(p=0.039)$ were significantly associated with poor outcome. The type of recanalization therapy, occurrence of pneumonia, and time to decompressive surgery were not significantly associated with poor outcome.

We combined the clinically relevant factors (1) higher patient age, (2) higher NIHSS score, (3) additional cerebral 


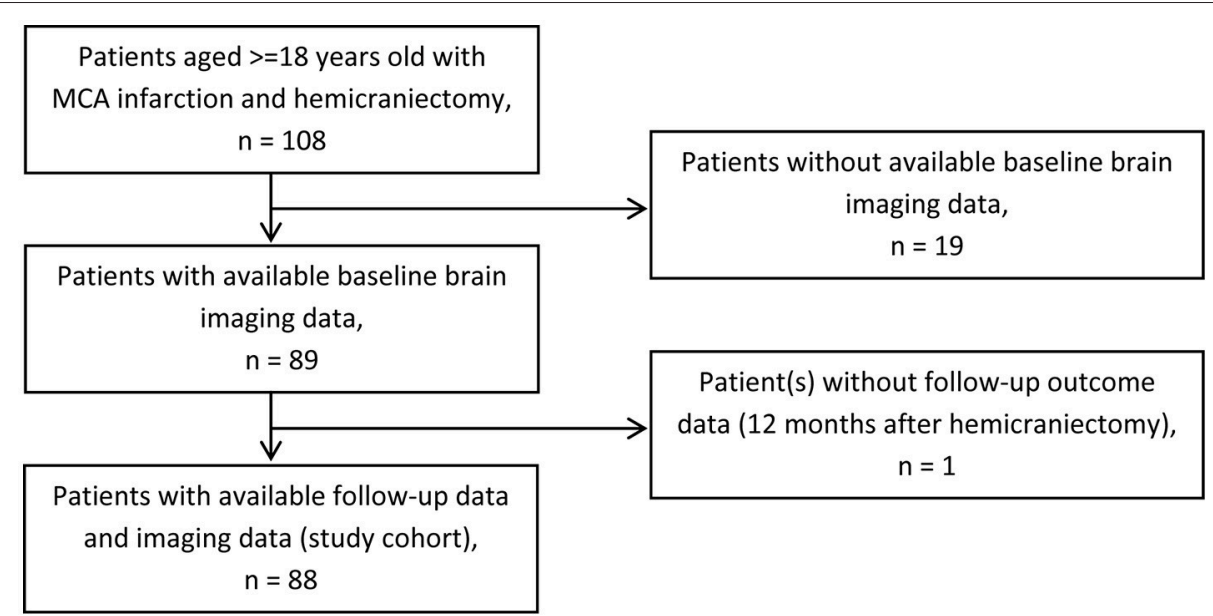

FIGURE 1 | Patient selection. Flow chart of patient selection for study cohort. MCA, middle cerebral artery.

TABLE 1 | Baseline characteristics, ICU treatment and functional outcome.

\begin{tabular}{|c|c|c|c|c|c|}
\hline & & All patients $(n=88)$ & $\begin{array}{l}\text { Patients with mRS } 0-4 \text { at } \\
12 \text { months }(n=44)\end{array}$ & $\begin{array}{l}\text { Patients with mRS 5-6 at } \\
12 \text { months }(n=44)\end{array}$ & $p$-value \\
\hline \multirow[t]{2}{*}{ Age (years) } & mean (SD) & $52.8(8.6)$ & $50.6(7.8)$ & $54.9(8.9)$ & 0.024 \\
\hline & median (IQR) & $53.5(46-59)$ & $49(45.25-58.25)$ & $55.5(47.25-60)$ & . \\
\hline Sex (female) & $n(\%)$ & $32(36.4)$ & $15(34.1)$ & 17 (38.6) & 0.658 \\
\hline Hemisphere (dominant) & $n(\%)$ & $47(53.4)$ & $23(52.3)$ & $24(54.5)$ & 0.831 \\
\hline NIHSS admission & median (IQR) & $19(15-32)$ & $17(14-20.75)$ & 22.5 (17.25-32) & $<0.001$ \\
\hline Atrial fibrillation & $n(\%)$ & $28(31.8)$ & $11(25.0)$ & $17(38.6)$ & 0.170 \\
\hline Sympton onset to DS (hours) & median (IQR) & $30.5(22-51.1)$ & $33(23.5-52.5)$ & $26.0(21-47.5)$ & 0.167 \\
\hline Osmotherapy & $n(\%)$ & $63(71.6)$ & $30(68.2)$ & $33(75.0)$ & 0.478 \\
\hline Barbiturates & $n(\%)$ & 19 (21.6) & $5(11.4)$ & $14(31.8)$ & 0.020 \\
\hline Hypothermia & $n(\%)$ & $74(84.1)$ & $36(81.8)$ & $38(51.4)$ & 0.560 \\
\hline Pneumonia & $n(\%)$ & $72(81.8)$ & 39 (88.6) & $33(75.0)$ & 0.097 \\
\hline Sepsis & $n(\%)$ & $18(20.5)$ & $7(15.9)$ & $11(25.0)$ & 0.290 \\
\hline Venous thrombosis & $n(\%)$ & $8(9.1)$ & $3(6.8)$ & $5(11.4)$ & 0.458 \\
\hline Renal replacement therapy & $n(\%)$ & $3(3.4)$ & $2(4.5)$ & $1(2.3)$ & 0.557 \\
\hline Hospital stay (days) & median (IQR) & 19 (13.3-25) & $20(17-25)$ & $16.5(8.25-24.75)$ & 0.038 \\
\hline In-hospital lethality & $n(\%)$ & $20(22.7)$ & . & . & NA \\
\hline mRS score at 12 months & median (IQR) & $4.5(3-6)$ & . & . & NA \\
\hline mRS score $0-3$ at 12 months & $n(\%)$ & $23(26.1)$ & . & . & NA \\
\hline mRS score $0-4$ at 12 months & $n(\%)$ & $44(50)$ & . & . & NA \\
\hline Lethality at 12 months & $n(\%)$ & $30(34.1)$ & . & $30(68.2)$ & NA \\
\hline
\end{tabular}

DS, decompressive surgery; ICU, intensive care unit; mRS, modified Rankin Scale; NA, not applicable.

infarction (ACA and/or PCA territory), (4) pneumonia, and (5) ipsilateral abnormal CVD into a multiple logistic regression model for prediction of poor outcome. During model simplification guided by the statistical AIC-criterion we dropped the factor pneumonia (Table 3). Higher patient age (OR 1.07, 95\%CI 1.01-1.14) and higher baseline NIHSS score (OR 3.42, 95\%CI 1.31-9.40) were independent predictors of poor outcome according to this model, but not ipsilateral abnormal CVD (OR 1.31, 95\%CI 0.47-3.67; $p=0.602)$.
Testing two other logistic regression models, substituting ipsilateral abnormal drainage with contralateral and any abnormal drainage, the model including the factor any abnormal venous drainage as numeric variable showed the best AIC value of all three models. A higher number of venous vessels with abnormal drainage was associated with poor functional outcome (OR 2.01, 95\%CI 0.80-5.39), but this effect was again not statistically significant. Contralateral abnormal CVD was also not an independent predictor of poor functional outcome at 12 months after hemicraniectomy. 
TABLE 2 | Imaging of space-occupying infarction and cerebral venous drainage.

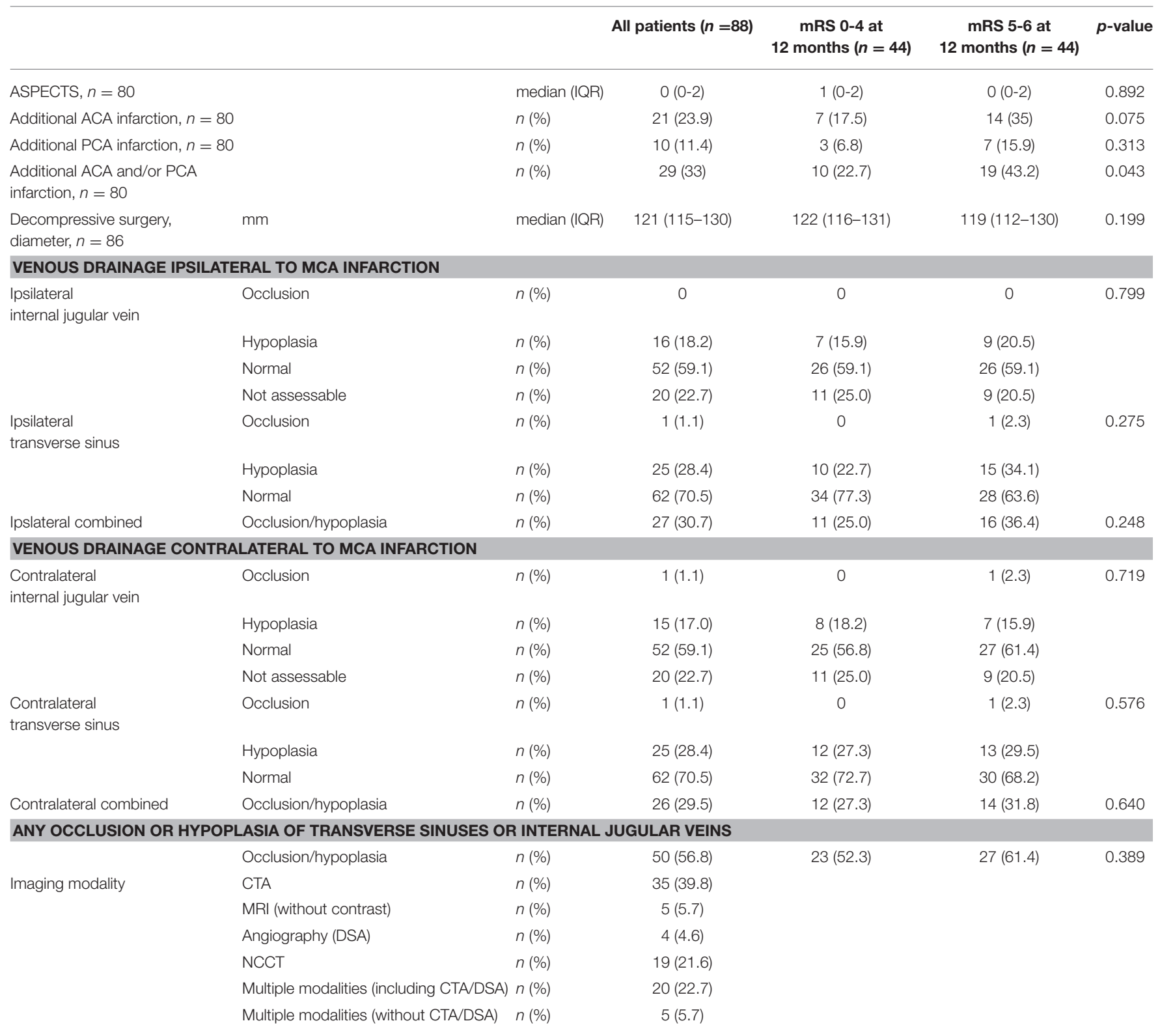

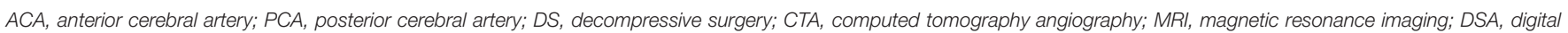
subtraction angiography; NCCT, non-contrast computed tomography; occl, occlusion.

Based on the observed proportions of poor functional outcome in patients with ipsilateral abnormal CVD and in patients with normal ipsilateral drainage in our cohort, in order to achieve a power of $80 \%$ for a 2 -sided Fisher's exact test with a significance level of $\alpha=0.05$, a sample size of 456 patients would be needed.

\section{DISCUSSION}

The primary hypothesis of our study was that an abnormal cerebral venous drainage ipsilateral to the side of malignant
MCA infarction in patients who underwent decompressive surgery would result in reduced venous drainage and consecutive venous congestion, more severe edema progression, and worse functional outcome compared to patients with normal ipsilateral venous drainage. However, in our study evaluating the CVD pattern in 88 patients with malignant MCA infarction who underwent hemicraniectomy, ipsilaterally abnormal venous drainage was not significantly associated with long-term functional outcome. Moreover, also contralaterally abnormal or any abnormal CVD were not predictive for poor functional outcome in this cohort. 


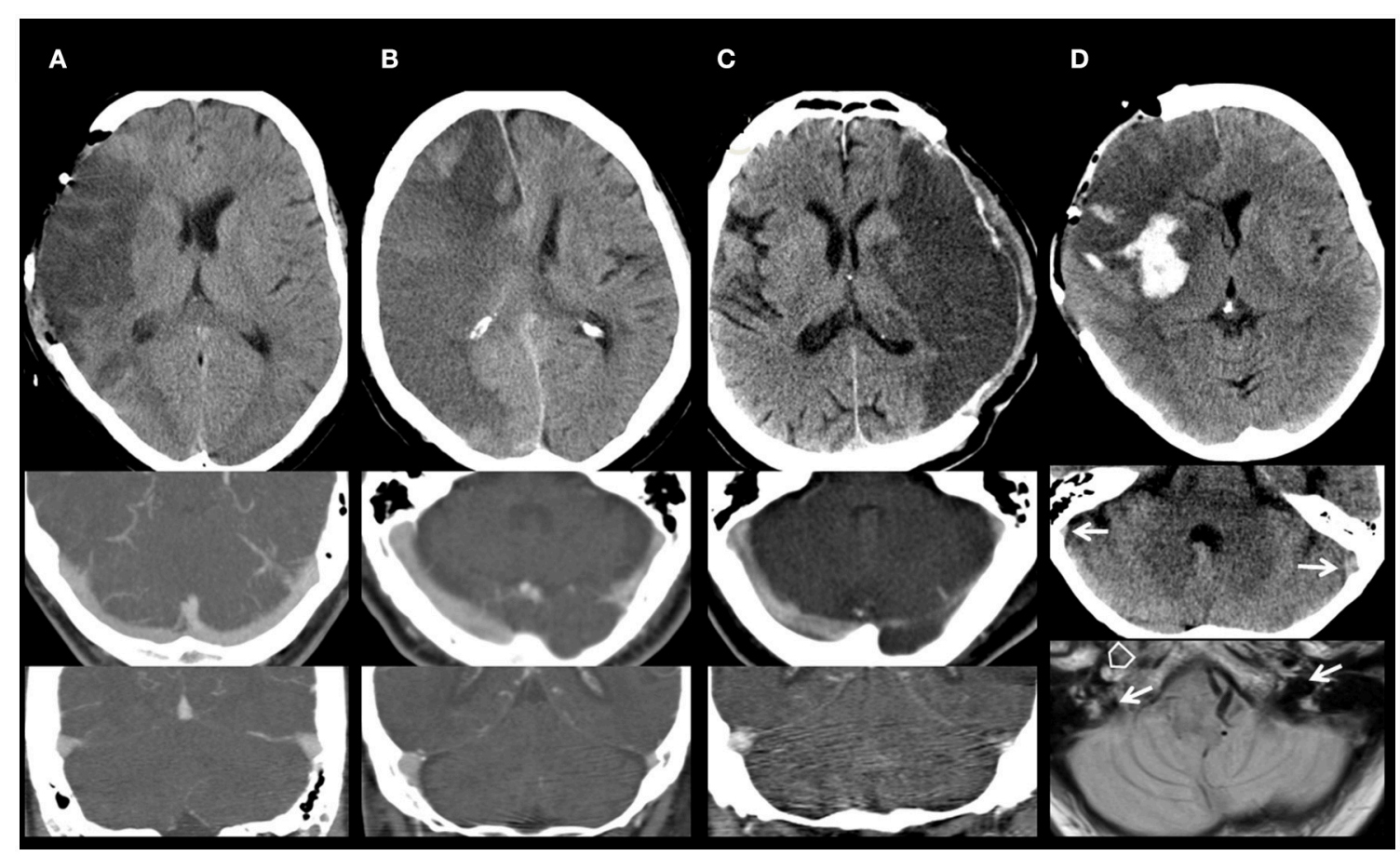

FIGURE 2 | Imaging of cerebral venous drainage. (A) Normal appearing venous drainage. Non-contrast computed tomography (NCCT) of a 60-year-old man with a large right middle cerebral artery (MCA) infarction after decompressive surgery. Symmetric venous drainage by both ipsilateral and contralateral transverse sinus and internal jugular veins (CTA). (B) Abnormal contralateral venous drainage. NCCT of a 66-year-old woman with a space-occupying right MCA and anterior cerebral artery infarction. Hypoplasia of the contralateral transverse sinus and internal jugular vein (CTA). (C) Abnormal ipsilateral venous drainage. NCCT of a 60-year-old man with a large left MCA infarction who underwent decompressive surgery. Hypoplasia of the ipsilateral transverse sinus and internal jugular vein (CTA). (D) Abnormal ipsilateral venous drainage. Imaging of a 62-year-old woman with a large right MCA infarction, after decompressive surgery (NCCT; Upper). Hypoplasia of the ipsilateral transverse sinus and internal jugular vein. NCCT (Middle): Asymmetry of the sigmoid sinus, hypoplastic right, dominant left (arrows). MRI (proton density; Lower): basal scan close to the foramen magnum. Open arrow: occluded right internal carotid artery (missing flow void). Filled arrows: dominant left internal jugular vein (IJV), hypoplastic right IJV.

TABLE 3 | Multiple logistic regression analysis for prediction of poor functional outcome (mRS scores 5-6).

\begin{tabular}{lccc}
\hline Predictor & OR & $\mathbf{9 5 - \% ~ C l ~}$ & $\boldsymbol{p}$-value \\
\hline Age & 1.07 & $1.01-1.14$ & 0.013 \\
NIHSS & 3.42 & $1.31-9.40$ & 0.012 \\
Additional ACA/PCA infarction & 2.24 & $0.83-6.31$ & 0.111 \\
Ipsilateral abnormal venous drainage & 1.31 & $0.47-3.67$ & 0.602 \\
\hline
\end{tabular}

$A C A$, anterior cerebral artery; PCA, posterior cerebral artery.

Baseline characteristics of patients included in our study were comparable to characteristics of patients with space-occupying MCA infarction in previous randomized hemicraniectomy trials $(6,7)$. Functional outcome was not directly comparable with earlier hemicraniectomy trials due to different inclusion criteria including patient age. We found an occlusion or hypoplasia of the transverse sinus in 30\% and of the internal jugular vein in $18 \%$ of patients. This is in line with earlier reports regarding human cerebral venous drainage (14-16).

The physiological role of CVD and its alterations in stroke patients have been studied rarely. After ischemic stroke by large artery occlusion the adjacent draining veins collapse due to reduced intra-vascular pressure and increased extravascular pressure. The reduced venous outflow might cause a redistribution of blood from the infarct core to the penumbra by collateral veins, a concept called cerebral blood steel phenomenon (17). The venous collateral status might therefore influence the development of ischemic brain edema. However, it is unclear whether patients with hypoplastic or aplastic venous sinuses have compensatory venous collaterals more frequently.

Some studies have focused on CVD and its impact on the prognosis of ischemic stroke. Using mono-phase CT angiography in stroke patients, impaired filling of deep and cortical veins in territories corresponding to the infarction was associated with poor functional outcome (18-20). Furthermore, asymmetric venous drainage in stroke patients was associated with larger areas of hypoperfusion and with worse clinical outcome (21). In a case series including 14 stroke patients, abnormal ipsilateral cranial venous drainage was present in 4 of 5 patients who developed fatal brain edema after MCA infarction (11). Although we could not detect a significant association between occlusion or hypoplasia of large venous segments and worse long-term functional outcome in patients with large MCA stroke, venous aplasia or hypoplasia were more frequently observed in patients with poor functional outcome. This was observed for all three 
composite variables (ipsilateral, contralateral, any abnormal cerebral venous drainage).

Similarly to previous studies, higher patient age and baseline stroke severity were independent predictors for poor functional outcome in our cohort (9). A score for stroke patients with MCA occlusion has been introduced recently to assess the probability of malignant brain edema (22). This Malignant Brain Edema Score incorporates (1) the baseline NIHSS Score, (2) the ASPECT Score, (3) the Clot Burden Score, grading the extent of arterial clotting, (23) (4) ipsilateral arterial collateral status, and (5) the large vessel recanalization status. Based on our study results, an incorporation of CVD state in prediction scores for long-term functional outcome after severe MCA stroke seems currently not justified.

About one-third of patients of the cohort had an additional infarction of the ACA/PCA territory, reflecting clinical practice to select patients for hemicraniectomy at our center during the study period. In previous studies, higher MCA infarction volume and occurrence of additional ACA/PCA infarction were shown to be independent predictors for poor outcome in patients with MCA infarction and hemicraniectomy $(24,25)$. Additional ACA/PCA infarction was more frequently observed in patients with poor outcome in our cohort, although not independently predictive for outcome, which is attributable in part to the number of patients included in our cohort. However, MCA infarction volume and/or additional infarctions need to be taken into account if hemicraniectomy is considered in patients with MCA infarction (26).

Our study has strengths and limitations. We report one of the largest case series of surgically treated patients with space-occupying MCA stroke who were evaluated for cerebral venous drainage. Furthermore, the selected stroke patients were routinely treated according to an institutional protocol during the observational period. The image analyses for the study were performed in a three reader consensus mode, limiting misinterpretation of imaging data.

Limitations of our study arise from the retrospective, singlecenter study design. Patients with MCA infarction were selected for the study cohort from a basic hemicraniectomy cohort by availability of imaging data, which was limited for the early time period considered, so selection bias may have influenced our results. The evaluation of functional outcome was performed retrospectively. However, we applied a definition of poor functional outcome as modified Rankin Scale scores of 5 or 6 which are both conditions that can reliably retrieved retrospectively. Another important limitation of our analysis is the size of our cohort. The regression analyses revealed associations of different patterns of abnormal CVD with poor functional outcome, but each not reaching statistical significance. Although the cohort was relatively large $(n=88)$, the number of patients included may have prevented the detection of statistically significant associations of abnormal CVD with outcome measures due to the lack of statistical power. Further studies with larger sample sizes should be undertaken to further evaluate the effect of CVD in patients with malignant MCA infarction.
Different imaging modalities with varying power for the detection of CVD patterns were used in our analyses. CTA was primarily used to detect arterial occlusions and not to evaluate venous drainage pattern, therefore interpretation of venous drainage using arterial-phase CTA is limited. As we used static evaluations and not a sequential or dynamic evaluation of CVD, the results should be interpreted with caution. The term "abnormal" drainage, used in our study for patients with aplastic, hypoplastic or occluded venous vessels, is based on anatomical and not on functional data, respectively. The combined dynamic and static contrast-enhanced MR venography has been previously been shown to accurately identify impaired cerebral venous flow and venous thrombosis $(27,28)$.

Furthermore, we used cerebral infarction patterns as imaging surrogate for impaired cerebral arterial influx, as angiographic data were available for only $67 \%$ of patients. Individual cerebral arterial flow patterns and intra-individual differences between both hemispheres might have contributed to edema progress and functional outcome in our cohort.

In conclusion, stroke patients with space-occupying MCA infarction who underwent decompressive surgery, an impaired cerebral venous drainage was observed more frequently in patients with poor functional outcome, but no significant associations of drainage patterns with clinical outcome measures were found. Prospective studies with larger sample sizes and using dynamic imaging modalities could further determine the role of venous drainage in patients with space-occupying MCA infarction.

\section{DATA AVAILABILITY STATEMENT}

The dataset used for analyses reported in this manuscript is not publicly available because consent statements of study patients and local data protection guidelines restrict its use to investigators of the Technische Universität Dresden, Germany, and their affiliates.

\section{AUTHOR CONTRIBUTIONS}

VP designed the study, acquired and interpreted data, and revised the manuscript for content. JG acquired and interpreted data and revised the manuscript for content. PK acquired and interpreted data and revised the manuscript for content. MK interpreted data, performed statistical analyses, and revised the manuscript for content. HR interpreted data and revised the manuscript for content. HS has designed the study, acquired and interpreted data, performed statistical analyses, and drafted the manuscript. All authors read and approved the final manuscript.

\section{FUNDING}

We acknowledge support by the Open Access Publication Funds of the SLUB/TU Dresden. The funding body had no impact on any aspect of the study and the content of the manuscript. 


\section{REFERENCES}

1. Hacke W, Schwab S, Horn M, Spranger M, De Georgia M, von Kummer R. 'Malignant' middle cerebral artery territory infarction: clinical course and prognostic signs. Arch Neurol. (1996) 53:309-15.

2. Frank JI. Large hemispheric infarction, deterioration, and intracranial pressure. Neurology (1995) 45:1286-90.

3. Vahedi K, Hofmeijer J, Juettler E, Vicaut E, George B, Algra A, et al. Early decompressive surgery in malignant infarction of the middle cerebral artery: a pooled analysis of three randomised controlled trials. Lancet Neurol. (2007) 6:215-22. doi: 10.1016/S1474-4422(07)70036-4

4. Jüttler E, Schwab S, Schmiedek P, Unterberg A, Hennerici M, Woitzik J, et al. Decompressive surgery for the treatment of malignant infarction of the middle cerebral artery (DESTINY): a randomized, controlled trial. Stroke (2007) 38:2518-25. doi: 10.1161/STROKEAHA.107.485649

5. Vahedi K, Vicaut E, Mateo J, Kurtz A, Orabi M, Guichard JP, et al. Sequentialdesign, multicenter,randomized, controlled trial of early decompressive craniectomy in malignant middle cerebral artery infarction (DECIMAL Trial). Stroke (2007) 38:2506-17. doi: 10.1161/STROKEAHA.107.485235

6. Hofmeijer J, Kappelle LJ, Algra A, Amelink GJ, van Gijn J, van der Worp HB. Surgical decompression for space-occupying cerebralinfarction (the Hemicraniectomy After Middle Cerebral Artery infarction with Lifethreatening Edema Trial [HAMLET]): a multicentre, open, randomised trial. Lancet Neurol. (2009) 8:326-33. doi: 10.1016/S1474-4422(09)70047-X

7. Jüttler E, Unterberg A, Woitzik J, Bösel J, Amiri H, Sakowitz OW, et al. Hemicraniectomy in older patients with extensive middle-cerebral-artery stroke. N Engl J Med. (2014) 370:1091-100. doi: 10.1056/NEJMoa1311367

8. Huttner HB, Schwab S. Malignant middle cerebral artery infarction: clinical characteristics, treatment strategies, and future perspectives. Lancet Neurol. (2009) 8:949-58. doi: 10.1016/S1474-4422(09)70224-8

9. Neugebauer H, Jüttler E. Hemicraniectomy for malignant middle cerebral arteryinfarction: current status and future directions. Int J Stroke (2014) 9:460-7. doi: 10.1111/ijs.12211

10. Schneider $H$, Krüger $P$, Algra A, Hofmeijer J, van der Worp HB, Jüttler E, et al. No benefits of hypothermia in patients treated with hemicraniectomy for large ischemic stroke. Int J Stroke (2017) 12:732-40. doi: 10.1177/1747493017694388

11. Yu W, Rives J, Welch B, White J, Stehel E, Samson D. Hypoplasia or occlusion of the ipsilateral cranial venous drainage is associated with early fatal edema of middle cerebral artery infarction. Stroke (2009) 40:3736-9. doi: 10.1161/STROKEAHA.109.563080

12. Pexman JH, Barber PA, Hill MD, Sevick RJ, Demchuk AM, Hudon ME, et al. Use of the Alberta Stroke Program Early CT Score (ASPECTS) for assessing CT scans in patients with acute stroke. Am J Neuroradiol. (2001) 22:1534-42.

13. Puetz V, Dzialowski I, Hill MD, Demchuk AM. The Alberta Stroke Program Early CT Score in clinical practice: what have we learned? Int J Stroke (2009) 4:354-64. doi: 10.1111/j.1747-4949.2009.00 337.x

14. Ito H, Kanno I, Iida H, Hatazawa J, Shimosegawa E, Tamura H, et al. Arterial fraction of cerebral blood volume in humans measured by positron emission tomography. Ann Nucl Med. (2001) 15:111-6. doi: 10.1007/BF02988600

15. Schaller B. Physiology of cerebral venous blood flow: from experimental data in animals to normal function in humans. Brain Res Rev. (2004) 46:243-60. doi: 10.1016/j.brainresrev.2004.04.005

16. Munuera J, Blasco G, Hernández-Pérez M, Daunis-I-Estadella P5, Dávalos A, Liebeskind DS, et al. Venous imaging-based biomarkers in acute ischaemic stroke. J Neurol Neurosurg Psychiatry (2017) 88:62-9. doi: 10.1136/jnnp-2016-314814
17. Pranevicius O, Pranevicius M, Pranevicius H, Liebeskind DS. Transition to collateral flow after arterial occlusion predisposes to cerebral venous steal. Stroke (2012) 43:575-9. doi: 10.1161/STROKEAHA.111.635037

18. Parthasarathy R, Kate M, Rempel JL, Liebeskind DS, Jeerakathil T, Butcher KS, et al. Prognostic evaluation based on cortical vein score difference in stroke. Stroke (2013) 44:2748-54. doi: 10.1161/STROKEAHA.113.001231

19. van den Wijngaard IR, Wermer MJ, Boiten J, Algra A, Holswilder G, Meijer FJ, et al. Cortical venous filling on dynamic computed tomographic angiography: a novel predictor of clinical outcome in patients with acute middle cerebral artery stroke. Stroke (2016) 47:762-7. doi: 10.1161/STROKEAHA.115.012279

20. Bhaskar S, Bivard A, Parsons M, Nilsson M, Attia JR, Stanwell P, et al. Delay of late-venous phase cortical vein filling in acute ischemic stroke patients: associations with collateral status. J. Cereb Blood Flow Metab. (2017) 37:67182. doi: 10.1177/0271678X16637611

21. Sharma VK, Yeo LL, Teoh HL, Shen L, Chan BP, Seet RC, et al. Internal cerebral vein asymmetry on follow-up brain computed tomography after intravenous thrombolysis in acute anterior circulation ischemic stroke is associated with poor outcome. J Stroke Cerebrovasc Dis. (2014) 23:e39-45. doi: 10.1016/j.jstrokecerebrovasdis.2013.08.007

22. Jo K, Bajgur SS, Kim H, Choi HA, Huh PW, Lee K. A simple prediction score system for malignant brain edema progression in large hemispheric infarction. PLoS ONE (2017) 12:e0171425. doi: 10.1371/journal.pone.0171425

23. Puetz V, Dzialowski I, Hill MD, Subramaniam S, Sylaja PN, Krol A, et al. Intracranial thrombus extent predicts clinical outcome, final infarct size and hemorrhagic transformation in ischemic stroke: the clot burden score. Int J Stroke (2008) 3:230-6. doi: 10.1111/j.1747-4949.2008.00221.x

24. Hecht N, Neugebauer H, Fiss I, Pinczolits A, Vajkoczy P, Jüttler E, et al. Infarct volume predicts outcome after decompressive hemicraniectomy for malignant hemispheric stroke. J Cereb Blood Flow Metab. (2018) 38:1096-103. doi: 10.1177/0271678X17718693

25. Kamran S, Salam A, Akhtar N, D'soza A, Shuaib A. Predictors of decompressive hemicraniectomy in malignant middle cerebral artery stroke. Neurosurg Rev. (2018) doi: 10.1007/s10143-018-0974-9. [Epub ahead of print].

26. Torbey MT, Bösel J, Rhoney DH, Rincon F, Staykov D, Amar AP, et al. Evidence-based guidelines for the management of large hemispheric infarction : a statement for health care professionals from the Neurocritical Care Society and the German Society for Neuro-intensive Care and Emergency Medicine. Neurocrit Care (2015) 22:146-64. doi: 10.1007/s12028-014-0085-6

27. Meckel S, Reisinger C, Bremerich J, Damm D, Wolbers M, Engelter S, et al. Cerebral venous thrombosis: diagnostic accuracy of combined, dynamic and static, contrast-enhanced 4D MR venography. Am. J. Neuroradiol. (2010) 31:527-35. doi: 10.3174/ajnr.A1869

28. Schnell S, Wu C, Ansari SA. Four-dimensional MRI flow examinations in cerebral and extracerebral vessels - ready for clinical routine? Curr. Opinions Neurol. (2016) 29:419-28. doi: 10.1097/WCO.0000000000000341

Conflict of Interest Statement: The authors declare that the research was conducted in the absence of any commercial or financial relationships that could be construed as a potential conflict of interest.

Copyright (C) 2018 Puetz, Gerber, Krüger, Kuhn, Reichmann and Schneider. This is an open-access article distributed under the terms of the Creative Commons Attribution License (CC BY). The use, distribution or reproduction in other forums is permitted, provided the original author(s) and the copyright owner(s) are credited and that the original publication in this journal is cited, in accordance with accepted academic practice. No use, distribution or reproduction is permitted which does not comply with these terms. 